\title{
Heavy-ions induced scintillation experiments
}

\author{
M. Lamotte, ${ }^{a, 1}$ G. De Izarra, ${ }^{a}$ C. Jammes ${ }^{a}$ \\ ${ }^{a}$ CEA, DEN, DER, Instrumentation, Sensors and Dosimetry Laboratory, Cadarache, F-13108 Saint-Paul- \\ Lez-Durance, France \\ E-mail: maxime.lamotte@cea.fr
}

\begin{abstract}
Aвstract: The French Alternative Energies and Atomic Energy Commission (CEA) is developing fission chambers relying on gas luminescence capable of high gamma signal rejection and selfdiagnostic capabilities allowing high dependability level. Scintillation yield of noble gases by energetic ions (average initial energy between $68 \mathrm{MeV}$ to $99 \mathrm{MeV}$ ) is evaluated for nuclear power reactor instrumentation application. Neutron interaction with fissile material can provide energetic heavy ions at a rate proportional to the neutron flux. The understanding of fission fragments as excitation source is required for noble gas scintillation neutron detectors. This work deals with evaluation of the optical signal heavy-ions induced in cold plasma. A dedicated experimental device (PSEG) is presented. Plutonium 238 is selected as a continuous source of heavy ions in order to avoid technological constraints of neutron flux tests, as a first step in the experimental validation process. Estimation of signal power is performed as well as Optical Emission Spectrum analysis. As expected, no Partial Local Thermodynamic Equilibrium is satisfied from experiments. The effect of pressure on the visible to infrared emission spectrum is analysed.
\end{abstract}

Keywords: Neutron detectors; Gaseous detectors; Plasma diagnostics - charged-particle spectroscopy

\footnotetext{
${ }^{1}$ Corresponding author.
} 


\section{Contents}

1 Introduction 1

2 Material and methods 1

3 Results and discussion 3

4 Conclusion 4

\section{Introduction}

Nuclear power reactors relies on available and dependable instrumentation for general control and protection [1]. The French Alternative Energies and Atomic Commission (CEA) proposes a new generation of radiation-hardened neutron detectors for reactor power monitoring, based on optical fission chambers. This alternative design requires no electrical power on the sensor side, while the signal may be transmitted with regular optical-fibres over tens of metres in harsh environment, ensuring a high dependability level. In such gaseous detectors, heavy ions with a high ionization power (initial charge between +15 and +22 ) are generated by fission reaction with a fissile coating. As the fission fragments slow-down in the rare gas by inelastic collisions, they generate deltarays (namely electrons) up to several $\mathrm{keV}$, bringing the gas' atoms to excited and ionised states. That ionised gas forms a cold plasma emitting de-excitation lines from spontaneous radiative decay of the excited gas' species, rising emission of photons in the visible-to-near-infrared (around $1 \mu \mathrm{m}$ )spectrum. The very high ionization potential of heavy ions in the gas ensures rejection of gamma-induced photoelectrons by level discrimination. This paper addresses the first milestones towards development of passive (in terms of voltage bias) neutron detection systems. A heavy-ion pumped cold plasma is produced in a dedicated plasma reactor and Optical Emission spectroscopy is performed.

\section{Material and methods}

A heavy ion-induced plasma reactor is designed, the PSEG, with its main goals being to mock up the slowing down of fission fragments in a future detector. The device is optimized for collecting Optical Emission Spectroscopy (OES) data to validate under development numerical plasma simulation code (Figure 1).

The excitation source consists of $1.8 \mathrm{mg}$ of ${ }^{238} \mathrm{PuO}_{2}$, an alpha particles emitter, with a $98 \%$ purity. Alpha particles have the same pair-production energy spent $\left(\mathrm{W}_{\mathrm{exc}}\right)$ on noble gases as that of heavy ions [2]. The plutonium is coated atop a planar stainless steel $\varnothing 18 \mathrm{~mm}$ sheet. The plutonium-coated surface is about $1.76 \mathrm{~cm}^{2}$ and $0.9 \mu \mathrm{m}$ thick. A total activity of $1 \mathrm{GBq}$ is expected, half of which will contribute to excite the gas, as $50 \%$ of the emitted ions will be stopped in the 


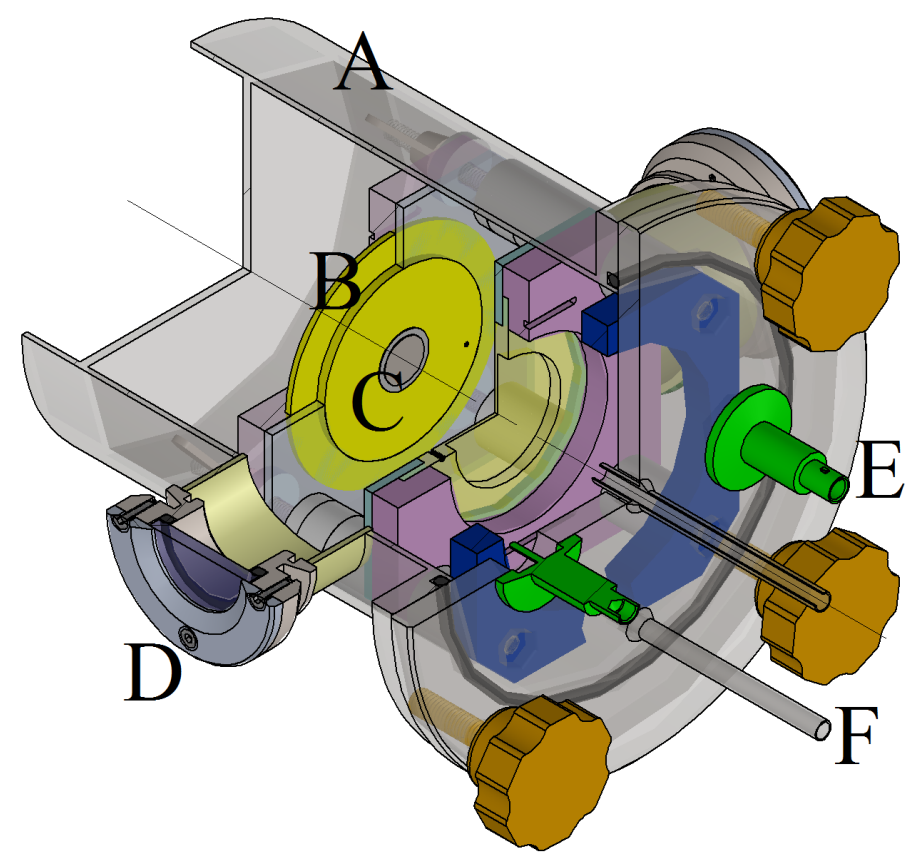

Figure 1. CAD model of PSEG. A: steel envelope, B: electrode, C: Plutonium layer D: view-port E: BNC connector F: gas inlet. Overall dimensions: $\varnothing 180 \mathrm{~mm}$ x $220 \mathrm{~mm}$

substrate. Given the main decay mode of ${ }^{238} \mathrm{PuO}_{2}$, one can estimate the energy flux deposited in the gas:

$$
\phi_{E}\left[e V . s^{-1}\right]=\frac{1}{2} \cdot A \cdot \int_{h=0}^{h=t} \int_{\theta=0}^{\pi} \int_{\phi=0}^{\pi / 2} E d h d \theta d \phi
$$

where in the half-infinite substrate of thickness $\mathrm{t}, \mathrm{h}$ is the height at which the alpha particle is generated at random angles $\theta$ and $\phi$ with a production rate $\mathrm{A}$.

Selecting a thin layer of radioactive material (with respect to the mean range of the alpha particles in the coating: $\approx 11 \mu \mathrm{m}$ ) is appealing for early development: the monoenergetic characteristic allows easier modelling of primary electron generation by means of semi-empirical simply differential ion-atom ionisation cross-sections. The increase of radioactive coating thickness only increases the emission rate at the coating interface in the limit of the range of the particles. A trade-off between energy deposition rate and alpha particles energy spectrum was performed as further thickness will screen alpha particles generated deeper in the coating. The rate of excited atom production in the cold plasma is roughly estimated by equation 2 relying on the Wexc-value of the target gas:

$$
Q_{\text {exc }}=\phi_{E} / W_{\text {exc }}
$$

where $W_{\text {exc }}$ is the energy required to produce an excited atom in a medium taking into account all phenomena such as primary electron excitation, electron collision cascade, heavy-ion excitation, etc...[3] $W_{\text {exc }}$ in rare gases excited by alpha particles and fission fragments has been measured by several authors and values about $53.8 \mathrm{eV}$ for Argon [4]. Fore pure rare gases, no substantial pressure effect on the W-values have been measured[7] Recalling equation 2, about 5.16E+13 argon atoms 
can be excited every second. The range of $5.56 \mathrm{MeV}$ alpha particles in argon at STP (Standard conditions for Temperature and Pressure) is about $37 \mathrm{~mm}$ [5]: a nuclear-excited plasma of argon at atmospheric pressure of the shape of a half sphere if the excitation source is punctual will therefore have a volume of about $100 \mathrm{~m} \ell$. This value was used reference for the design of PSEG. Two high purity silica windows ensure a $93 \%$ transmission of the optical signal between the ultraviolet to near-infrared range. The $\emptyset 30 \mathrm{~mm}$ windows are mounted on machined stainless flanges, sealed with silicone o'rings, and sized for operations between rough vacuum to 7 atm filling. Materials used to manufacture PSEG imply slow degassing properties (polymers are avoided) in order to prevent pollution of the gas during and out of operation. A pair of high-voltage feedtrough with BNC connectors are welded on the lid of PSEG for a possible polarization of electrodes. Helium and argon fillings from standard industrial-welding cylinders are injected at various pressure in the $1.1 \ell$ PSEG. The typical Arcal Prime industrial argon contains less than $100 \mathrm{ppm}$ of pollutants. A Princeton Instrument SP2558 spectrometer with a focal length of $50 \mathrm{~cm}$, Silver-coated mirrors and CCD Pixis camera with resolution of $1340 \times 100$ pixels record emission spectra with 600 grooves $/ \mathrm{mm}$ $1 \mu \mathrm{m}$ blazed grating. Near-infrared pictures of the plasma helps to locate specific regions of the nuclear-excited gas as no radiation is visible by the naked eye. A standard CCD general purpose camera without infrared filter captures an image of the plasma in 3 minutes after background subtraction.

\section{Results and discussion}

Figure 2 shows a photography of a 2 and 4 atm argon plasma excited by ${ }^{238} \mathrm{PuO}_{2}$ alpha source. The impact of pressure on the shape and intensity of the plasma is clearly visible, as well as the range of the alpha particles and plasma's volume. The upper luminous part of the picture is a reflection of the light by the polished stainless steel electrodes.

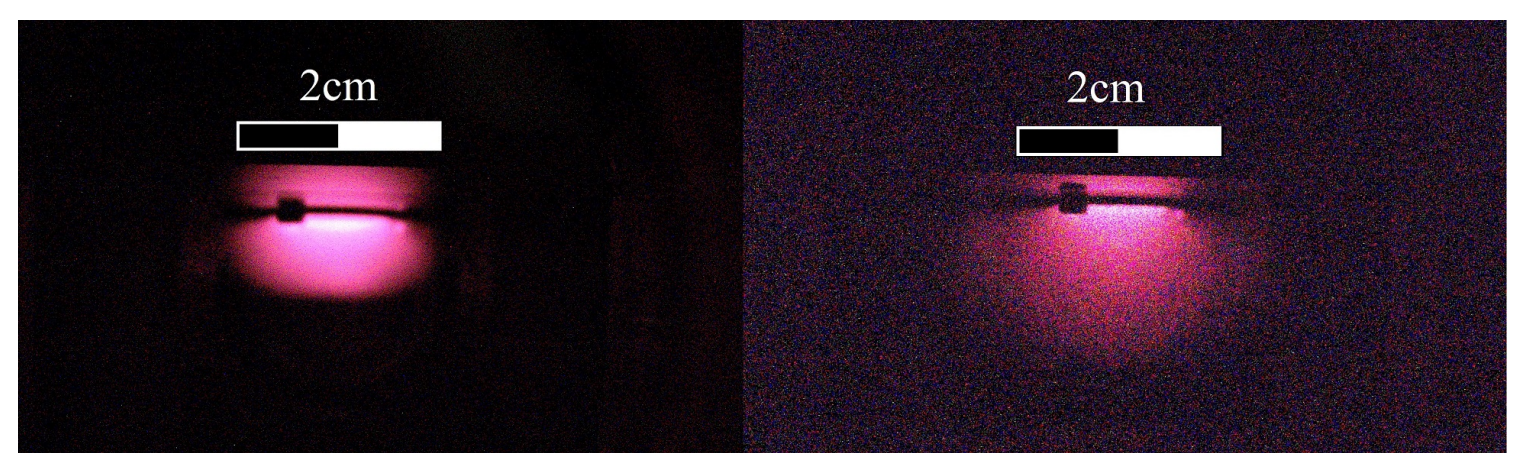

Figure 2. Picture of an alpha-particle excited Argon plasma. a) 4 atm b) 2 atm .

Preliminary spectra of the unfocused plasma were acquired with 3 minutes exposures between 300 and $1500 \mathrm{~nm}$. The source of excitation was located $20 \mathrm{~cm}$ away from the spectrometer's entrance slit opened of $10 \mu \mathrm{m}$. Pressures ranging between 0.1 and 4 atm were set in PSEG after pumpingdegassing cycles to exhaust any molecular contamination between fillings. A strong emission spectrum in the near-infrared was recorded in the case of argon, and no pollutant molecular bands (eg: from nitrogen or water) were found. 


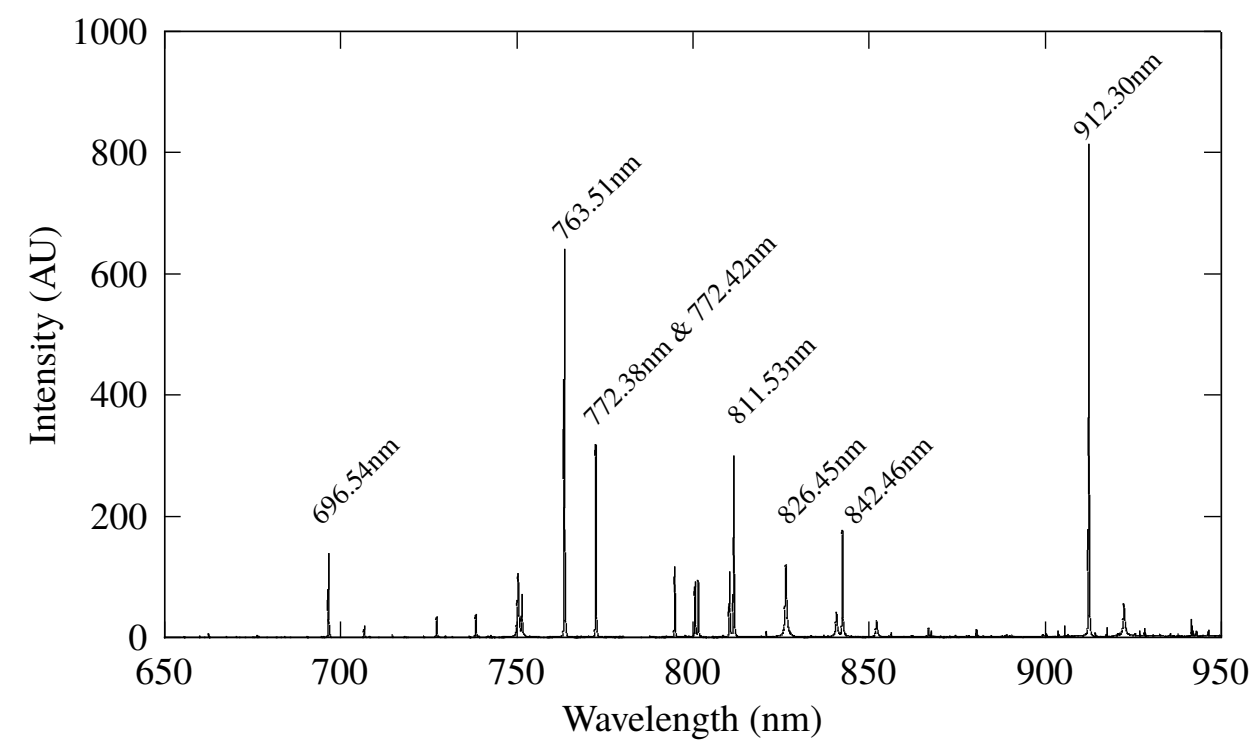

Figure 3. Emission spectrum of a 2 atm alpha particle-excited argon plasma acquired in 20 minutes. No argon ionized species' emission lines are detected. Strong Ar-I emission lines are labelled.

The absence of continuum component on the spectra seems to indicate radiative recombinations and bremsstrahlung effects are negligible, hence translating the very low ionisation rate of the studied plasma. Long acquisitions of $12 \mathrm{~h}$ did not show any continuum radiation either but the background infrared signal removed after dark shots.

Assuming a Local Thermodynamic Equilibrium (LTE), a linear relationship between $\ln \left(\frac{\lambda_{i j} I_{i j}}{h c A_{i j} g_{j}}\right)$ and the upper levels' energies $E_{j}$ can be plotted [6], where I are the line intensities, $\lambda$ their respective transition wavelengths, A their spontaneous de-excitation coefficient. A least-square line fitting to data points may indicate LTE conditions were met and the plasma is optically thin if no points deviate from the line.

Boltzmann plots where produced as a way to estimate the population of Ar I excitation state and possibly retrieve an excitation temperature from the slope of line fit to data points.

Given the sparse data on argon excited species mostly relying on the lower energy levels ${ }^{2} \mathrm{P}_{10}$ to ${ }^{2} \mathrm{P}_{1}$, no clear tendency can be cleared: no transitions involving upper energy levels above $13.47 \mathrm{eV}$ could be detected even with long ( $2 \mathrm{~h}$ ) acquisitions. The tendency line, with a coefficient of determination as low as 0.02, displays a positive slope, incompatible with LTE hypothesis thus, no plasma temperature could be estimated using Boltzmann plots. As expected for cold plasma created in such way, LTE conditions do not hold.

\section{Conclusion}

A heavy-ion cold plasma reactor has been designed for noble gas scintillation studies. Pictures of argon plasma excited by $5.56 \mathrm{MeV}$ alpha particles where obtained using an infrared-filter free CCD camera. Range of ions and general shape of the cold plasma has been assessed. An 


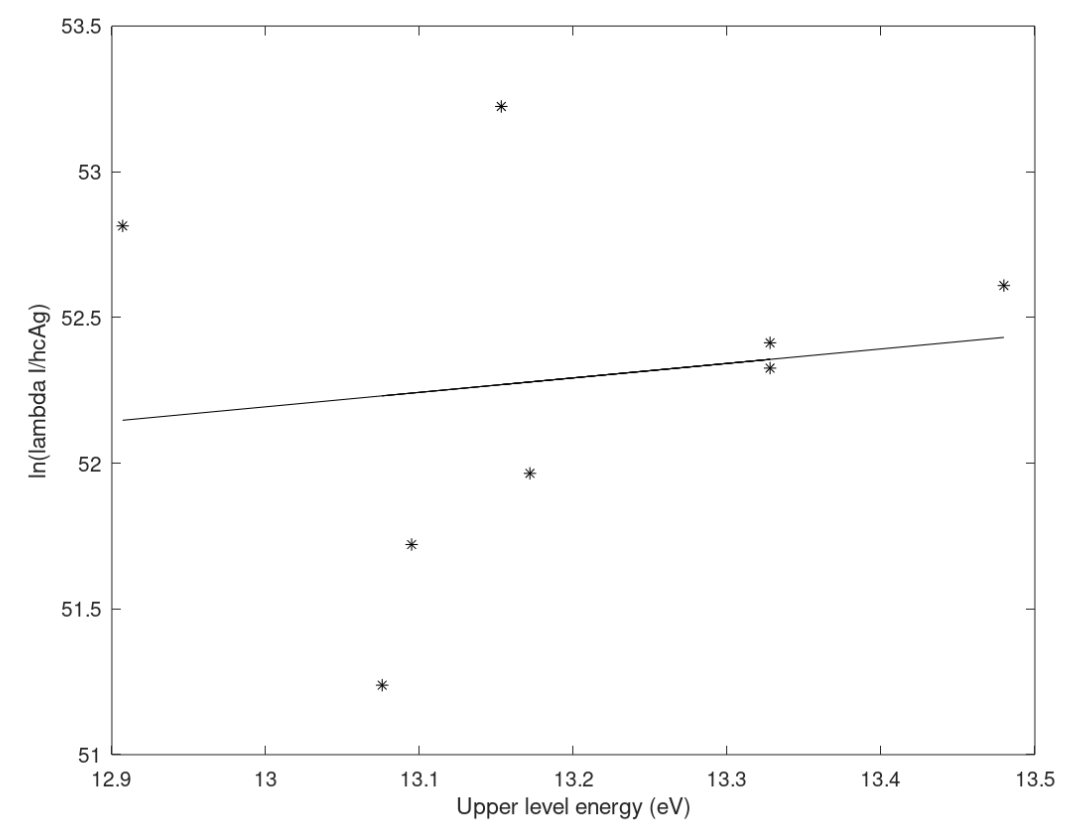

Figure 4. Boltzmann plot of an alpha particle-excited argon plasma of $0.5 \mathrm{~atm}$.

Optical Emission Spectroscopy analysis of Argon ion excited cold-plasma was performed at ranging pressures. Lower energy levels seems to be the most populated as no emission line from decays above level ${ }^{2} \mathrm{P}_{1}$ were detected at any pressure. The pressure of the cold plasma impacts the shape and the emission line intensity. Boltzmann plots based on Argon-I emission lines were produced. No Local Thermodynamic Equilibrium holds. A collisional-radiative model will be developed to understand and simulate processes involved in heavy-ion excited high pressure noble gas cold plasmas.

\section{References}

[1] Jammes, C et al Neutron flux monitoring system of the French GEN-IV SFR: Assessment of diverse solutions for in-vessel detector installation, Nucl. Eng. Des. 270 (2014)

[2] Giesen, U. and Beck, J., New measurements of W-values for protons and alpha particles, Radiat. Prot. Dosim. 161 (2014)

[3] Platzman, R.L Total ionization in gases by high-energy particles: An appraisal of our understanding, Appl. Radiat. Isot. 10 (1961)

[4] Walters, R Excitation of gases by fission fragments, PhD thesis, Univ. of Florida (1973)

[5] Walters, R Stopping-Power \& Range Tables for Electrons, Protons, and Helium Ions. NIST Standard Reference Database 124, NIST (2017)

[6] Kunze, H-J Introduction to Plasma Spectroscopy, Elsevier (2009)

[7] Parks, J.E et al Ionization of the Noble Gases by Protons: Jesse Effects as a Function of Pressure, J.Chem.Phys 57 (1972) 